\title{
Nonlocal fractional functional differential equations with measure of noncompactness in Banach space
}

\author{
Junfei Cao $^{1} \cdot$ Qian Tong $^{1} \cdot$ Xianyong Huang ${ }^{1}$
}

Received: 5 October 2014/ Accepted: 17 April 2015/Published online: 9 May 2015

(c) The Author(s) 2015. This article is published with open access at Springerlink.com

\begin{abstract}
In this paper, we are concerned with the following fractional functional differential equations with nonlocal initial conditions in Banach space

$$
\begin{gathered}
\mathrm{D}^{\alpha} x(t)=A x(t)+f\left(t, x(t), x_{t}\right), \quad t \in[0, T], \\
x(0)=\phi+g(x) .
\end{gathered}
$$

By virtue of the theory of measure of noncompactness associated with Darbo's fixed point theorem, upon making some suitable assumptions, some existence results of mild solutions are obtained. Moreover the results obtained are utilized to study the existence of solutions to fractional parabolic equations as an illustrative example to show the practical usefulness of the analytical results.
\end{abstract}

Keywords Fractional functional differential equation . Nonlocal initial condition - Hausdorff measure of noncompactness · Mild solution · Darbo's fixed point theorem

\section{Introduction}

In this paper, we are concerned with the nonlocal initial value problem

$$
\begin{aligned}
\mathrm{D}^{\alpha} x(t) & =A x(t)+f\left(t, x(t), x_{t}\right), \quad t \in[0, T], \\
x(0) & =\phi+g(x),
\end{aligned}
$$

where $A$ is the infinitesimal generator of a strongly

Junfei Cao

jfcaomath@163.com

1 Department of Mathematics, Guangdong University of Education, Guangzhou 510310, People's Republic of China continuous semigroup of bounded linear operators $T(t)$ in a separable Banach space $X$,

$f:[0, T] \times X \times C \rightarrow X, \quad g: L^{p}([0, T], X) \rightarrow X$,

are given $X$-valued functions. The fractional derivative is understood in the Riemann-Liouville sense. The aim of this paper is to study the existence of mild solutions for the fractional functional differential Eq. (1.1) in a separable Banach space. The technique used here is the measure of noncompactness associated with Darbo's fixed point theorem.

The fractional derivative is understood in the RiemannLiouville sense. The origin of fractional calculus goes back to Newton and Leibnitz in the seventieth century. One observes that fractional order can be very complex in viewpoint of mathematics and they have recently proved to be valuable in various fields of science and engineering. In fact, one can find numerous applications in electrochemistry, electromagnetism, viscoelasticity, biology and hydrogeology. For example space-fractional diffusion equations have been used in groundwater hydrology to model the transport of passive tracers carried by fluid flow in a porous medium $[1,2]$ or to model activator-inhibitor dynamics with anomalous diffusion [3]. For details, see [47] and the references therein.

Differential equations of fractional order have appeared in many branches of physics and technical sciences [8,9]. It has seen considerable development in the last decade, see [3-29] and the references therein. Recently, the existence and uniqueness problem for various fractional differential equations were considered by Ahmad [10], Bhaskarc[11], Lakshmikantham and Leela[12] et al. The nonlocal Cauchy problem was considered by Anguraj, Karthikeyan and N'Guérékata [13], and the importance of nonlocal initial 
conditions in different fields has been discussed in $[6,7]$ and the references therein.

The nonlocal problem (1.1) was motivated by physical problems. Indeed, the nonlocal initial condition $x(0)=$ $\phi+g(x)$ can be applied in physics with better effect than the classical initial condition $x(0)=\phi$. For this reason, the problem (1.1) has gotten considerable attention in recent years, see [30-32] and the references therein. See also [33$35]$ and the references therein for recent generalizations of problem (1.1) to various kinds of differential equations.

To the best of our knowledge, the existence of mild solutions for the fractional functional differential Eq. (1.1) with nonlocal initial conditions using the theory of measure of noncompactness is a subject that has not been treated in the literature. Our purpose in this paper is to establish some results concerning the existence of mild solutions for equations that can be modeled in the form (1.1) by virtue of the theory of measure of noncompactness associated with Darbo's fixed point theorem. Upon making some appropriate assumptions, some sufficient conditions for the existence of mild solutions for the fractional functional differential Eq. (1.1) are given. It is worthwhile mentioning that the cases of $T(t)$ or $f$ compact and of $f$ Lipschitz are special cases of our conditions. Also we hope that the concept of measure of noncompactness considered here may be a stimulant for further investigations concerning solutions of fractional differential equations of other types.

The rest of this paper is organized as follows. In "Notations, definitions and auxiliary facts" section, we give some notations, definitions and auxiliary facts. "Main results" section contains the main results of this paper with two existence theorems. An example is given to illustrate our results in "Applications" section.

\section{Notations, definitions and auxiliary facts}

Let $(X,\|\cdot\|)$ be a real separable Banach space. Denote by $C([0, T], X)$ the space of $X$-valued continuous functions on $[0, T]$ and by $L^{p}([0, T], X)$ the space of $X$-valued measurable functions on $[0, T]$ with

$\int_{0}^{T}\|x(t)\|^{p} \mathrm{~d} t<\infty$

provided with norm

$$
\|x\|_{p}=\left(\int_{0}^{T}\|x(t)\|^{p} \mathrm{~d} t\right)^{\frac{1}{p}} .
$$

Let $r$ be a given positive real number, if $x:[-r, T] \rightarrow X$, define $x_{t} \in C([-r, 0], X)$ by

$$
x_{t}(\theta)=x(t+\theta), \text { for }-r \leq \theta \leq 0,
$$

and denote

$$
\|x\|_{C}=\sup \{\|x(t)\|: t \in[-r, 0]\}, \text { for } x_{t} \in C([-r, 0], X) .
$$

We need some basic definitions and properties of the fractional calculus theory which are used further in this paper.

Definition 2.1 [36] The fractional integral of order $\alpha>0$ with the lower limit $t_{0}$ for a function $f$ is defined as

$$
I^{\alpha} f(t)=\frac{1}{\Gamma(\alpha)} \int_{t_{0}}^{t}(t-s)^{\alpha-1} f(s) \mathrm{d} s, \quad t>t_{0}, \quad \alpha>0,
$$

provided the right-hand side is pointwise defined on $\left[t_{0}, \infty\right)$, where $f$ is an abstract continuous function and $\Gamma(\alpha)$ is the Gamma function [36].

Definition 2.2 [36] Riemann-Liouville derivative of order $\alpha>0$ with the lower limit $t_{0}$ for a function $f$ : $\left[t_{0}, \infty\right) \rightarrow \mathbb{R}$ can be written as

$$
\begin{gathered}
D_{t}^{\alpha} f(t)=\frac{1}{\Gamma(n-\alpha)} \frac{\mathrm{d}^{\mathrm{n}}}{\mathrm{d} t^{n}} \int_{t_{0}}^{t}(t-s)^{-\alpha} f(s) \mathrm{d} s, \\
t>t_{0}, \quad n-1<\alpha<n .
\end{gathered}
$$

The first and maybe the most important property of Riemann-Liouville fractional derivative is that for $\mathrm{t}>t_{0}$ and $\alpha>0$, we have

$D_{t}^{\alpha}\left(I^{\alpha} f(t)\right)=f(t)$,

which means that Riemann-Liouville fractional differentiation operator is a left inverse to the Riemann-Liouville fractional integration operator of the same order $\alpha$.

Let $Y$ be a Banach space, for bounded set $B \subset Y$, the Hausdorff's measure of noncompactness $\chi_{Y}$ is defined by $\chi_{Y}(B)=\inf \{r>0, B$ can be covered by finite number of balls with radii $r\}$.

In this paper, we denote $\chi$ by the Hausdorff's measure of noncompactness of $X$ and denote $\chi_{p}$ by the Hausdorff's measure of noncompactness of $L^{p}([0, T], X)$. To discuss the problem in this paper, we need the following lemmas.

Lemma 2.1 [37] Let $B, C \subset Y$ be bounded, the following properties are satisfied

(1) $B$ is precompact if and only if $\chi_{Y}(B)=0$;

(2) $\chi_{Y}(B)=\chi_{Y}(\bar{B})=\chi_{Y}(\operatorname{conv} B)$, where $\bar{B}$ and $\operatorname{conv} B$ mean the closure and convex hull of $B$, respectively;

(3) $\chi_{Y}(B) \leq \chi_{Y}(C)$ when $B \subseteq C$;

(4) $\chi_{Y}(B+C) \leq \chi_{Y}(B)+\chi_{Y}(C)$, where $B+C=\{x$ $+y: x \in B, y \in C\}$; 
(5) $\quad \chi_{Y}(B \cup C) \leq \max \left\{\chi_{Y}(B), \chi_{Y}(C)\right\}$;

(6) $\chi_{Y}(\lambda B)=|\lambda| \chi_{Y}(B)$ for any $\lambda \in \mathbb{R}$;

(7) If the map $Q: D(Q) \subseteq Y \rightarrow Z$ is Lipschitz continuous with constant $k$, then

$\chi_{Z}(Q B) \leq k \chi_{Y}(B)$,

for any bounded subset $B \subseteq D(Q)$, where $Z$ is a Banach space;

(8) $\chi_{Y}(B)=\inf \left\{d_{Y}(B, C): C \subseteq Y\right.$ be precompact $\}$

$=\inf \left\{d_{Y}(B, C): C \subseteq Y\right.$ be finite valued $\}$, where $d_{Y}(B, C)$ means Hausdorff distance between $B$ and $C$ in $Y$;

(9) If $\left\{W_{n}\right\}_{n=1}^{\infty}$ is a decreasing sequence of bounded closed nonempty subsets of $Y$ and

$\lim _{n \rightarrow \infty} W_{n}=0$,

then $\cap_{n=1}^{\infty} W_{n}$ is nonempty and compact in $Y$. The map $Q: W \subseteq Y \rightarrow Y$ is said to be a $\chi_{Y}$-contraction if there exists a positive constant $k<1$ such that

$\chi_{Y}(Q(C)) \leq k \chi_{Y}(C)$,

for any bounded closed subset $C \subseteq W$.

In 1955, Darbo [38] proved the fixed point property for $\alpha$-set contraction (i.e., $\alpha(S(A)) \leq k \alpha(A)$ with $k \in[0,1]$ ) on a closed, bounded and convex subset of Banach spaces. Since then many interesting works have appeared. For example, in 1972, Sadovskii [39] proved the fixed point property for condensing functions (i.e., $\alpha(S(A))<\alpha(A)$ with $\alpha(A) \neq 0$ ) on closed, bounded and convex subset of Banach spaces. It should be noted that any $\alpha$-set contraction is a condensing function, but the converse is not true (see [40]). In 2007, Hajji and Hanebaly [41] proved the existence of a common fixed point for commuting mappings satisfying

$\alpha(S(A)) \leq k \sup _{i \in I}\left(\alpha\left(T_{i}(A)\right)\right), \alpha(S(A))<\sup _{i \in I}\left(\alpha\left(T_{i}(A)\right), \alpha(A)\right)$,

where $\alpha$ is the measure of noncompactness on a closed, bounded and convex subset $\Omega$ of a locally convex space $X$, $T_{i}$ and $S$ are continuous functions from $\Omega$ to $\Omega$ with $T_{i}$, and in addition, are affine or linear. Furthermore, for every $i \in I, T_{i}$ are equal to the identity function, moreover the obtain in particular Darbo's (see [38]) as well as Sadovskii's (see [39]) fixed point theorems, which are used to study the existence of solutions of one equation. Recently, Hajji [42] present common fixed point theorems for commuting operators which generalize Darbo's and Sadovskii's fixed point theorems, furthermore, as examples and applications, they study the existence of common solutions of equations in Banach spaces using measure of noncompactness. Our purpose in this paper is to establish some results concerning the existence of mild solutions for equations that can be modeled in the form (1.1) by virtue of the theory of measure of noncompactness associated with Darbo's fixed point theorem.

Lemma 2.2 ([37], Darbo-Sadovskii) If $W \subseteq Y$ is bounded closed and convex, the continuous map $Q: W \rightarrow$ $W$ is a $\chi_{Y}$-contraction, then $Q$ has at least one fixed point in $W$.

We call $B \subset L([0, T], X)$ uniformly integrable if there exists $\eta \in L\left([0, T], \mathbb{R}^{+}\right)$such that

$\|u(s)\| \leq \eta(s)$, for all $u \in B$ and a.e. $s \in[0, T]$.

Lemma 2.3 [43] If $\left\{u_{n}\right\}_{n=1}^{\infty} \in L([0, T], X)$ is uniformly integrable, then $t \rightarrow \chi\left(\left\{u_{n}(t)\right\}_{n=1}^{\infty}\right)$ is measurable and

$\chi\left(\left\{\int_{0}^{t} u_{n}(s) \mathrm{d} s\right\}_{n=1}^{\infty}\right) \leq \int_{0}^{t} \chi\left(\left\{u_{n}(s)\right\}_{n=1}^{\infty}\right) \mathrm{d} s$.

Lemma 2.4 [44] Let $B \subset C([0, T], X)$ be bounded and equicontinuous on $[0, T]$. Then

$\chi_{p}=\left(\int_{0}^{T} \chi^{p}(B(t)) \mathrm{d} t\right)^{\frac{1}{p}}$

where

$B(t)=\{u(t): u \in B\} \subset X$.

A $C_{0}$ semigroup $T(t)$ is said to be equicontinuous if

$t \rightarrow\{T(t) x: x \in B\}$

is equicontinuous for all bounded set $B$ in $X$ and $\mathrm{t}>0$. It is known that the analytic semigroup is equicontinuous.

The following lemma is obvious.

Lemma 2.5 If the semigroup $T(t)$ is equicontinuous, $\eta \in L\left([0, T], \mathbb{R}^{+}\right)$, then the set

$\left\{\frac{1}{\Gamma(\alpha)} \int_{0}^{t}(t-s)^{\alpha-1} T(t-s) u(s) \mathrm{d} s:\|u(s)\| \leq \eta(s)\right\}$,

for a.e. $s \in[0, T]$, is equicontinuous for all $t \in[0, T]$.

Proof Note that,

$$
\begin{aligned}
& \| \frac{1}{\Gamma(\alpha)} \int_{0}^{t+h}(t+h-s)^{\alpha-1} T(t+h-s) u(s) \mathrm{d} s \\
& \quad-\frac{1}{\Gamma(q)} \int_{0}^{t}(t-s)^{\alpha-1} T(t-s) u(s) \mathrm{d} s \| \\
& \leq \frac{1}{\Gamma(\alpha)} \| \int_{0}^{t}\left[(t+h-s)^{\alpha-1}-(t-s)^{\alpha-1}\right] \\
& \quad T(t+h-s) u(s) \mathrm{d} s \| \\
& \quad+\frac{1}{\Gamma(\alpha)}\left\|\int_{t}^{t+h}(t+h-s)^{\alpha-1} T(t+h-s) u(s)\right\| \mathrm{d} s
\end{aligned}
$$




$$
\begin{aligned}
& +\frac{1}{\Gamma(\alpha)}\left\|\int_{0}^{t}(t-s)^{\alpha-1}[T(t+h-s)-T(t-s)] u(s)\right\| \mathrm{d} s \\
= & I+I I+I I I,
\end{aligned}
$$

where

$$
\begin{aligned}
& I=\frac{1}{\Gamma(\alpha)} \| \int_{0}^{t}\left[(t+h-s)^{\alpha-1}-(t-s)^{\alpha-1}\right] \\
& T(t+h-s) u(s) \mathrm{d} s \| \\
& I I=\frac{1}{\Gamma(\alpha)}\left\|\int_{t}^{t+h}(t+h-s)^{\alpha-1} T(t+h-s) u(s)\right\| \mathrm{d} s \\
& I I I=\frac{1}{\Gamma(\alpha)}\left\|\int_{0}^{t}(t-s)^{\alpha-1}[T(t+h-s)-T(t-s)] u(s)\right\| \mathrm{d} s .
\end{aligned}
$$

Estimating the terms on the right-hand side of (2.1) yields

$$
\begin{aligned}
I \leq & \frac{M}{\Gamma(\alpha)} \int_{0}^{t}\left|(t+h-s)^{\alpha-1}-(t-s)^{\alpha-1}\right| \eta(s) \mathrm{d} s \\
\leq & \frac{M}{\Gamma(\alpha)} \int_{0}^{t-\varepsilon}\left[(t-s)^{\alpha-1}-(t+h-s)^{\alpha-1}\right] \eta(s) \mathrm{d} s \\
& +\frac{M}{\Gamma(q)} \int_{t-\varepsilon}^{t}(t-s)^{\alpha-1} \eta(s) \mathrm{d} s \\
= & I^{\prime}+I I^{\prime}
\end{aligned}
$$

where

$$
\begin{aligned}
& I^{\prime}=\frac{M}{\Gamma(\alpha)} \int_{0}^{t-\varepsilon}\left[(t-s)^{\alpha-1}-(t+h-s)^{\alpha-1}\right] \eta(s) \mathrm{d} s \\
& I I^{\prime}=\frac{M}{\Gamma(q)} \int_{t-\varepsilon}^{t}(t-s)^{\alpha-1} \eta(s) \mathrm{d} s,
\end{aligned}
$$

with

$$
M=\sup \{\|T(t)\|: t \in[0, T]\} .
$$

It follows from the assumption of $\eta(s)$ that $I^{\prime} \rightarrow 0$ as $h \rightarrow 0$. Using Hölder inequality, one obtains $I I^{\prime} \rightarrow 0$ as $h \rightarrow 0$ and $\varepsilon \rightarrow 0$.

For $I I$, one has

$$
\begin{aligned}
I I & \leq \frac{1}{\Gamma(\alpha)} \int_{t}^{t+h}(t+h-s)^{\alpha-1}\|T(t+h-s) u(s)\| \mathrm{d} s \\
& \leq \frac{M}{\Gamma(\alpha)} \int_{t}^{t+h}(t+h-s)^{\alpha-1} \eta(s) \mathrm{d} s \rightarrow 0 \text { as } h \rightarrow 0 .
\end{aligned}
$$

As to III, one gets

$$
\begin{aligned}
I I I \leq & \frac{1}{\Gamma(\alpha)}\left\|\int_{0}^{t-\varepsilon}(t-s)^{\alpha-1}[T(t+h-s)-T(t-s)] u(s)\right\| \mathrm{d} s \\
& +\frac{1}{\Gamma(\alpha)}\left\|\int_{t-\varepsilon}^{t}(t-s)^{\alpha-1}[T(t+h-s)-T(t-s)] u(s)\right\| \mathrm{d} s
\end{aligned}
$$

$$
\begin{aligned}
\leq & \frac{1}{\Gamma(\alpha)} \int_{0}^{t-\varepsilon}(t-s)^{\alpha-1}\left\|T\left(\frac{h}{2}+\frac{t+h-s}{2}\right)-T\left(\frac{t-s}{2}\right)\right\| \\
& \left\|T\left(\frac{t-s}{2}\right) u(s)\right\| \mathrm{d} s \\
& +\frac{2 M}{\Gamma(\alpha)} \int_{t-\varepsilon}^{t}(t-s)^{\alpha-1} \eta(s) \mathrm{d} s \\
\leq & \frac{M}{\Gamma(\alpha)} \int_{0}^{t-\varepsilon}(t-s)^{\alpha-1} \| T\left(\frac{h}{2}+\frac{t+h-s}{2}\right) \\
& -T\left(\frac{t-s}{2}\right) \| \eta(s) \mathrm{d} s+\frac{2 M}{\Gamma(\alpha)} \int_{t-\varepsilon}^{t}(t-s)^{\alpha-1} \eta(s) \mathrm{d} s \\
= & I^{\prime \prime}+I I^{\prime \prime},
\end{aligned}
$$

where

$$
\begin{aligned}
I^{\prime \prime}= & \frac{M}{\Gamma(\alpha)} \int_{0}^{t-\varepsilon}(t-s)^{\alpha-1} \| T\left(\frac{h}{2}+\frac{t+h-s}{2}\right) \\
& -T\left(\frac{t-s}{2}\right) \| \eta(s) \mathrm{d} s \\
I I^{\prime \prime}= & \frac{2 M}{\Gamma(\alpha)} \int_{t-\varepsilon}^{t}(t-s)^{\alpha-1} \eta(s) \mathrm{d} s .
\end{aligned}
$$

Using the assumption that $T(t)$ is equicontinuous in $X$, integrating with $s \rightarrow \eta(s) \in L\left([0, T], \mathbb{R}^{+}\right)$, one sees that $I^{\prime \prime} \rightarrow 0$ as $h \rightarrow 0$. From the assumption of $\eta(s)$ and Hölder inequality, it is easy to see that $I I^{\prime \prime} \rightarrow 0$ as $h \rightarrow 0$ and $\varepsilon \rightarrow 0$. Therefore, the family of functions

$$
\left\{\frac{1}{\Gamma(\alpha)} \int_{0}^{\cdot}(\cdot-s)^{\alpha-1} T(\cdot-s) u(s) \mathrm{d} s:\|u(s)\| \leq \eta(s)\right\},
$$

is equicontinuous.

\section{Main results}

In this section, we use the measure of noncompactness of $L^{p}([0, T], X)$ to consider the following functional differential equations of fractional order $0<\alpha>1$ when $g$ is continuous in the norm of $L^{p}([0, T], X)$

$$
\mathrm{D}^{\alpha} x(t)=A x(t)+f\left(t, x(t), x_{t}\right), \quad t \in[0, T], \quad x(0)=\phi+g(x) .
$$

Eq. (3.1) will be considered under the following assumptions:

(H) The $C_{0}$-semigroup $\{T(t)\}_{t \geq 0}$ generated by $A$ is equicontinuous;

$\left(\mathbf{H}_{2}\right)$

(1) $f:[0, T] \times X \times C([-r, 0], X) \rightarrow X$ satisfies the Carathéodory-type condition, i.e., $f(\cdot, x, \varphi):[0, T] \rightarrow X$ 
is measurable for all $(t, x, \varphi) \in[0, T] \times X \times$ $C([-r, 0], X)$ and $f(t, \cdot): X \times C([-r, 0], X) \rightarrow X$ is continuous for a.e. $t \in[0, T]$;

(2) there exists $d_{2}, e_{2} \in L^{p}\left([0, T], \mathbb{R}^{+}\right)$such that for all $(t, x, \varphi) \in[0, T] \times X \times C([-r, 0], X)$

$\|f(t, x, \varphi)\| \leq d_{2}(t)\left(\|x\|+\|\varphi\|_{C}\right)+e_{2}(t)$

(3) there exists $c_{2} \in L^{q}\left([0, T], \mathbb{R}^{+}\right)$such that for a.e. $t, s \in[0, T]$ and any bounded subset $D_{1} \subseteq X$, $D_{2} \subseteq C([-r, 0], X)$

$$
\begin{aligned}
\chi\left(T(t) f\left(s, D_{1}, D_{2}\right)\right) \leq & c_{2}(t) \\
& \left(\chi\left(D_{1}\right)+\sup _{\theta \in[-r, 0]} \chi\left(D_{2}(\theta)\right)\right),
\end{aligned}
$$

where

$D_{2}(\theta)=\left\{v(\theta): v \in D_{2}\right\}$ and $\frac{1}{p}+\frac{1}{q}=1 ;$

$\left(\mathbf{H}_{3}\right)$

(1) The function $g: L^{p}([0, T], X) \rightarrow X$ is continuous;

(2) there exist positive constants $d_{1}, e_{1}$ such that $\|g(x)\| \leq d_{1}\|x\|_{p}+e_{1}$, for any $x \in L^{p}([0, T], X) ;$

(3) there exists a positive constant $c_{1}$ such that for any $B \subset C([0, T], X)$ which is bounded and equicontinuous on $[0, T]$,

$\chi(T(t) g(B)) \leq c_{1} \chi_{p}(B)$, for any a.e. $t \in[0, T]$

$\left(\mathbf{H}_{4}\right)$

$M d_{1} T^{\frac{1}{p}}+\frac{4 M T^{\alpha}\left\|d_{2}\right\|_{p}}{\Gamma(\alpha)}\left(\frac{p-1}{p \alpha-1}\right)^{\frac{p-1}{p}}<1$.

Definition 3.1 A continuous function $x:[-r, T] \rightarrow X$ satisfying the integral equation

$$
\begin{aligned}
x(t)= & T(t)(x(0)-g(x))+\frac{1}{\Gamma(\alpha)} \\
& \int_{0}^{t}(t-\eta)^{\alpha-1} T(t-\eta) f\left(\eta, x(\eta), x_{\eta}\right) \mathrm{d} \eta,
\end{aligned}
$$

is called a mild solution for Eq.(3.1).

Now, we are prepared to state and prove our main theorems of this section.

Theorem 3.1 Let $\left.\left(H_{1}\right)-\left(H_{4}\right)\right)$ be satisfied. Then Eq.(3.1) has at least one mild solution whenever

$$
T^{\frac{1}{p}}\left(c_{1}+\frac{2\left\|c_{2}\right\|_{q} T^{\alpha-\frac{1}{p}}}{\Gamma(\alpha)}\left(\frac{p-1}{p \alpha-1}\right)^{\frac{p-1}{p}}\right)<1 .
$$

Proof For each $k \in \mathbb{N}$, denote by

$$
\begin{aligned}
B_{k}=B_{k}\left(L^{p}([-r, T], X)\right) & =\left\{x \in L^{p}([-r, T], X):\|x(s)\|\right. \\
& \leq k, s \in[-r, T]\} .
\end{aligned}
$$

Obviously $B_{k} \subset L^{p}([-r, T], X)$ is uniformly integrable, closed and convex. For each $x \in B_{k}$, the restriction of $x$ on $[0, T]$ denoted by $\left.x\right|_{[0, T]}$ is an element of $B_{k}\left(L^{p}([0, T], X)\right)$. For simplicity, we also write $g\left(\left.x\right|_{[0, T]}\right)$ as $g(x)$.

Define $F: L^{p}([-r, T], X) \rightarrow L^{p}([-r, T], X)$ by $F=F_{1}+$ $F_{2}$, where

$$
\left(F_{1} x\right)(t)= \begin{cases}\phi(t), & t \in[-r, 0], \\ T(t)(x(0)-g(x)), & t \in[0, T],\end{cases}
$$

$\left(F_{2} x\right)(t)$

$$
= \begin{cases}0, & t \in[-r, 0], \\ \frac{1}{\Gamma(\alpha)} \int_{0}^{t}(t-\eta)^{\alpha-1} T(t-\eta) f\left(\eta, x(\eta), x_{\eta}\right) \mathrm{d} \eta, & t \in[0, T] .\end{cases}
$$

First, we show that $F$ is well defined.

If $t \in[-r, 0]$, then

$\|F x(t)\| \leq\|\phi\|_{C}$,

and if $t \in[0, T]$, one has

$$
\begin{aligned}
& \|F x(t)\| \leq\left\|F_{1} x(t)+F_{2} x(t)\right\| \\
\leq & M(\|\phi(0)\|+\|g(x(0))\|) \\
& +\frac{1}{\Gamma(\alpha)} \int_{0}^{t}\left\|(t-\eta)^{\alpha-1} T(t-\eta) f\left(\eta, x(\eta), x_{\eta}\right)\right\| \mathrm{d} \eta \\
\leq & M\left[\|\phi(0)\|+d_{1}\|x\|_{p}+e_{1}\right. \\
& \left.+\frac{1}{\Gamma(\alpha)} \int_{0}^{t}(t-\eta)^{\alpha-1}\left[d_{2}(\eta)\left(\|x\|+\|x\|_{C}\right)+e_{2}(\eta)\right] \mathrm{d} \eta\right]
\end{aligned}
$$$$
\leq M\left(\|\phi(0)\|+d_{1}\|x\|_{p}+e_{1}\right)+\frac{M}{\Gamma(\alpha)}\left(\int_{0}^{t}(t-\eta)^{\frac{(\alpha-1) p}{p-1}} \mathrm{~d} s\right)^{\frac{p-1}{p}}
$$$$
\left(\int_{0}^{t}\left(d_{2}(\eta)\left(\|x\|+\|x\|_{C} \|\right)+e_{2}(\eta)\right)^{p} \mathrm{~d} \eta\right)^{\frac{1}{p}}
$$$$
\leq M\left(\|\phi(0)\|+d_{1}\|x\|_{p}+e_{1}\right)+\frac{M}{\Gamma(\alpha)}\left(\frac{p-1}{p \alpha-1}\right)^{\frac{p-1}{p}} T^{\alpha-\frac{1}{p}}
$$$$
\left(\int_{0}^{t}\left(d_{2}(\eta)\left(\|x\|+\|x\|_{C} \|\right)+e_{2}(\eta)\right)^{p} \mathrm{~d} \eta\right)^{\frac{1}{p}}
$$$$
\leq M\left(\|\phi(0)\|+d_{1} T^{\frac{1}{p}} k+e_{1}\right)+\frac{2 M}{\Gamma(\alpha)}\left(\frac{p-1}{p \alpha-1}\right)^{\frac{p-1}{p}}
$$

$$
T^{\alpha-\frac{1}{p}}\left[2\left\|d_{2}\right\|_{p} T^{\frac{1}{p}} k+\left\|e_{2}\right\|_{p}\right] .
$$


Thus, one has

$$
\begin{aligned}
\|F x(t)\| \leq & \max \left\{\|\phi\|_{C}, M\left(\|\phi(0)\|+d_{1} T^{\frac{1}{p}} k+e_{1}\right)\right. \\
& \left.+\frac{2 M T^{\alpha-\frac{1}{p}}}{\Gamma(\alpha)}\left(\frac{p-1}{p \alpha-1}\right)^{\frac{p-1}{p}}\left[2\left\|d_{2}\right\|_{p} T^{\frac{1}{p}} k+\left\|e_{2}\right\|_{p}\right]\right\} .
\end{aligned}
$$

Thus, we conclude that $F x$ exists.

Second, we show that there is a $k \in \mathbb{N}$ such that $F\left(B_{k}\right) \subseteq B_{k}$.

Suppose contrary that for each $k \in \mathbb{N}$ there is $x^{k} \in B_{k}$ and $t^{k} \in[-r, T]$ such that

$\left\|F x\left(t^{k}\right)\right\|>k$.

If $t^{k} \in[-r, 0]$, then

$k \leq\left\|F x\left(t^{k}\right)\right\| \leq\left\|\phi\left(t^{k}\right)+g\left(x\left(t^{k}\right)\right)\right\| \leq\|\phi\|_{C}+d_{1} T^{\frac{1}{p}} k+e_{1}$,

and if $t^{k} \in[0, T]$, one has

$$
\begin{aligned}
k \leq & \left\|F x\left(t^{k}\right)\right\| \leq\left\|F_{1} x\left(t^{k}\right)+F_{2} x\left(t^{k}\right)\right\| \\
\leq & M(\|\phi(0)\|+\|g(x(0))\|) \\
& +\frac{1}{\Gamma(\alpha)} \int_{0}^{t^{k}}\left\|\left(t^{k}-\eta\right)^{\alpha-1} Q\left(t^{k}-\eta\right) f\left(\eta, x(\eta), x_{\eta}\right)\right\| \mathrm{d} \eta \\
\leq & M\left[\|\phi(0)\|+d_{1}\|x\|_{p}+e_{1}\right. \\
& \left.+\frac{1}{\Gamma(\alpha)} \int_{0}^{t}(t-\eta)^{\alpha-1}\left[d_{2}(\eta)\left(\|x\|+\|x\|_{C}\right)+e_{2}(\eta)\right] \mathrm{d} \eta\right] \\
\leq & M\left(\|\phi(0)\|+d_{1} T^{\frac{1}{p}} k+e_{1}\right)+\frac{2 M}{\Gamma(\alpha)}\left(\frac{p-1}{p \alpha-1}\right)^{\frac{p-1}{p}} \\
& T^{\alpha-\frac{1}{p}\left[\left\|d_{2}\right\|_{p} T^{\frac{1}{p}} k+\left\|e_{2}\right\|_{p}\right] .}
\end{aligned}
$$

Divided by $k$ on both sides of (3.2), one has

$$
1 \leq M d_{1} T^{1}+\frac{2 M T^{\alpha}\left\|d_{2}\right\|_{p}}{\Gamma(\alpha)}\left(\frac{p-1}{p \alpha-1}\right)^{\frac{p-1}{p}},
$$

which contradicts the hypotheses $\left(H_{4}\right)$. Therefore, there is a $k \in \mathbb{N}$ such that $F\left(B_{k}\right) \subseteq B_{k}$.

From now on, we will restrict $F$ on such $B_{k}$.

Third, we will verify that $F$ is a $\chi_{C}$-contraction.

To this end, from the hypothesises $\left(H_{2}\right)(1)$ and (3), one can prove that $F$ is continuous by the continuity of $g$ and of the operator $f$. The hypothesis $\left(H_{1}\right)$ and Lemma 2.5 imply that $F B_{k} \subset C([0, T], X)$ is bounded and equicontinuous on $[0, T]$, so is $\operatorname{conv}\left(F B_{k}\right)$. As $X$ is separable, from Lemma 2.1 and Lemma 2.3-2.5 for any $B \subset \operatorname{conv}\left(F B_{k}\right)$, one has

$$
\begin{aligned}
\chi(F B(t)) \leq & \chi\left(T(t)\left(x_{0}-g(B)\right)\right) \\
& +\chi\left(\frac{1}{\Gamma(\alpha)} \int_{0}^{t}(t-\eta)^{\alpha-1} T(t-\eta) f\left(\eta, B(\eta), B_{\eta}\right)\right.
\end{aligned}
$$

$$
\begin{aligned}
\leq & \chi\left(T(t)\left(x_{0}-g(B)\right)\right) \\
& +\frac{1}{\Gamma(\alpha)} \int_{0}^{t} \chi\left((t-\eta)^{\alpha-1} T(t-\eta) f\left(\eta, B(\eta), B_{\eta}\right) \mathrm{d} \eta\right. \\
\leq & \chi\left(T(t)\left(x_{0}-g(B)\right)\right)+\frac{1}{\Gamma(\alpha)} \\
& \int_{0}^{t}(t-\eta)^{\alpha-1} \chi\left(T(t-\eta) f\left(\eta, B(\eta), B_{\eta}\right) \mathrm{d} \eta\right. \\
\leq & c_{1} \chi_{p}(B)+\frac{2}{\Gamma(\alpha)} \int_{0}^{t}(t-\eta)^{\alpha-1} c_{2}(\eta) \chi(B) \mathrm{d} \eta \\
\leq & \left(c_{1}+\frac{2\left\|c_{2}\right\|_{q} T^{\alpha-\frac{1}{p}}}{\Gamma(\alpha)}\left(\frac{p-1}{p \alpha-1}\right)^{\frac{p-1}{p}}\right) \chi_{p}(B),
\end{aligned}
$$

for a.e. $t \in[0, T]$, where

$B(t)=\{x(t): x \in B\} \subseteq X, B_{t}=\left\{x_{t}: x \in B\right\} \subseteq C([-r, 0], X)$.

By Lemma 2.3, this implies that

$$
\chi_{p}(F B) \leq T^{\frac{1}{p}}\left(c_{1}+\frac{2\left\|c_{2}\right\|_{q} T^{\alpha-\frac{1}{p}}}{\Gamma(\alpha)}\left(\frac{p-1}{p \alpha-1}\right)^{\frac{p-1}{p}}\right) \chi_{p}(B) .
$$

Note that, by Lemma 2.4, the inequality (3.3) may not remain valid in the case of $B \subset B_{k}$ as $B_{k}$ is not equicontinuous on $[0, T]$. So one must look for another closed convex and bounded subset of $L^{p}([0, T], X)$ such that $F$ is a $\chi_{p}$-contraction on it.

Let

$U=L^{p}-\operatorname{conv}\left(F B_{k}\right)$,

where $L^{p}-$ conv means closure of convex hull in $L^{p}([0, T], X)$. Then

$F U \subset U$ as $F B_{k} \subset B_{k}$,

and $B_{k}$ is closed and convex in $L^{p}([0, T], X)$. For any closed subset $V \subset U$, let

$B=V \cap \operatorname{conv}\left(F B_{k}\right)$.

Then

$V=L^{p}-\operatorname{cl}(B)$

where $L^{p}-\mathrm{cl}$ means closure in $L^{p}([0, T], X)$. Furthermore $F V \subset L^{p}-\operatorname{cl}(F B)$

as $F$ is continuous on $L^{p}([0, T], X)$. By (3.3) this implies that

$$
\begin{aligned}
\chi_{p}(F V) & \leq \chi_{p}\left(L^{p}-\operatorname{cl}(F B)\right)=\chi_{p}(F B) \\
& \leq T^{\frac{1}{p}}\left(c_{1}+\frac{2\left\|c_{2}\right\|_{q} T^{\alpha-\frac{1}{p}}}{\Gamma(\alpha)}\left(\frac{p-1}{p \alpha-1}\right)^{\frac{p-1}{p}}\right) \chi_{p}(B)
\end{aligned}
$$




$$
\leq T^{\frac{1}{p}}\left(c_{1}+\frac{2\left\|c_{2}\right\|_{q} T^{\alpha-\frac{1}{p}}}{\Gamma(\alpha)}\left(\frac{p-1}{p \alpha-1}\right)^{\frac{p-1}{p}}\right) \chi_{p}(V) .
$$

Since

$$
T^{\frac{1}{p}}\left(c_{1}+\frac{2\left\|c_{2}\right\|_{q} T^{\alpha-\frac{1}{p}}}{\Gamma(\alpha)}\left(\frac{p-1}{p \alpha-1}\right)^{\frac{p-1}{p}}\right)<1,
$$

so $F: U \rightarrow U$ is a continuous $\chi_{p}$-contraction. By DarboSadovskii's fixed point theorem, there is a fixed point $x$ of $F$ on $B_{k}$, which is a mild solution of the Eq. (3.1). This completes the proof due to Lemma 2.2.

Remark 3.1 Clearly the conclusion of Theorem 3.1 remains valid if the hypotheses $\left(H_{2}\right)(3)$ and $\left(H_{4}\right)$ (3) are replaced by the following $\left(H_{2}\right)\left(3^{\prime}\right)$ and $\left(H_{4}\right)\left(3^{\prime}\right)$, respectively: $\left(H_{2}\right)\left(3^{\prime}\right)$ There exists $c_{2} \in L^{q}\left([0, T], \mathbb{R}^{+}\right)$such that

$\chi\left(f\left(t, D_{1}, D_{2}\right)\right) \leq \frac{c_{2}(t)}{M}\left(\chi\left(D_{1}\right)+\sup _{\theta \in[-r, 0]} \chi\left(D_{2}(\theta)\right)\right)$,

for a.e. $[0, T]$ and any bounded subset $D_{1} \subseteq X$, $D_{2} \subseteq C([-r, 0], X)$;

$\left(H_{4}\right)\left(3^{\prime}\right)$ There exists a positive constant $c_{1}$ such that for any $B \subset C([0, T], X)$ which is bounded and equicontinuous on $[0, T]$,

$\chi(g(B)) \leq c_{1} \chi_{p}(B) / M$, for any a. e. $t \in[0, T]$.

Remark 3.2 The hypothesis $\left(H_{2}\right)$ (3') $\left(H_{4}\right)\left(3^{\prime}\right)$ holds if $T(t)$ is compact or $f(g)$ is the sum of compact and Lipschitz functions with constant $c_{2}(s)=M\left(c_{1}=M\right)$.

If $X$ is a Hilbert space, and $\phi$ is a proper, convex and lower semicontinuous function from $X$ into $(-\infty,+\infty)$, then its subdifferential $\partial \Phi$ is $m$-accretive. Let $A=\partial \Phi$ then $A$ generates an equicontinuous nonlinear contraction semigroup (cf. [45, 46]). From above we can get the following existence result.

Corollary 3.1 If $X$ is a separable Hilbert space, the hypotheses $\left(H_{2}\right)-\left(H_{4}\right)$ are true, and $A=\partial \Phi$ with $\phi$ is proper, convex and lower semicontinuous from $X$ into $(-\infty,+\infty)$. Then the nonlocal Eq. (3.1) has at least one integral solution provided that

$T^{\frac{1}{p}}\left(c_{1}+\frac{2\left\|c_{2}\right\|_{q} T^{\alpha-\frac{1}{p}}}{\Gamma(\alpha)}\left(\frac{p-1}{p \alpha-1}\right)^{\frac{p-1}{p}}\right)<1$.

Let us now formulate an existence result when $\mathrm{g}$ is uniformly bounded.

Theorem 3.2 Assume that $\left(H_{1}\right),\left(H_{3}\right)$ and $\left(H_{4}\right)$ are true with $d_{1}=0$, and $f$ satisfies $\left(H_{2}\right)(2)$, (3). In addition, suppose that there exists $\theta \in L^{p}\left([0, T], \mathbb{R}^{+}\right)$, an increasing function $\Omega: \mathbb{R}^{+} \rightarrow \mathbb{R}^{+}$such that

$$
\|f(t, x, \varphi)\| \leq \theta(t) \Omega\left(\|x\|+\|\varphi\|_{C}\right),
$$

for $(t, x, \varphi) \in[0, T] \times X \times C([-r, 0], X)$. Then Eq.(3.1) has at least one mild solution whenever

$$
\begin{aligned}
& T^{\frac{1}{p}}\left(c_{1}+\frac{3\left\|c_{2}\right\|_{q} T^{q-1}}{\Gamma(q)}\right)<1, \\
& M\left(\|\phi\|+e_{1}\right)+\frac{M T^{\alpha-\frac{1}{p}}\|\theta(s)\|_{p} \Omega\left(2 T^{\frac{1}{p}} k\right)}{\Gamma(\alpha)}\left(\frac{p-1}{p \alpha-1}\right)^{\frac{p-1}{p}} \leq k .
\end{aligned}
$$

Proof Define

$W_{0}=\left\{x \in L^{p}([0, T], X):\|x(t)\| \leq k\right.$ for a.e. $\left.t \in[0, T]\right\}$.

For any $x \in W_{0}$,

$$
\begin{aligned}
\|F x(t)\| \leq & \left\|F_{1} x(t)+F_{2} x(t)\right\| \\
\leq & M(\|\phi(0)\|+\|g(x(0))\|)+\frac{1}{\Gamma(\alpha)} \\
& \int_{0}^{t}\left\|(t-\eta)^{\alpha-1} T(t-\eta) f\left(\eta, x(\eta), x_{\eta}\right)\right\| \mathrm{d} \eta \\
\leq & M\left[\|\phi(0)\|+e_{1}+\frac{1}{\Gamma(\alpha)}\right. \\
& \left.\int_{0}^{t}(t-\eta)^{\alpha-1} \theta(\eta) \Omega\left(\|x\|+\|x\|_{C}\right) \mathrm{d} \eta\right] \\
\leq & M\left(\|\phi(0)\|+e_{1}\right)+\frac{M T^{\alpha-\frac{1}{p}}}{\Gamma(\alpha)}\left(\frac{p-1}{p \alpha-1}\right)^{\frac{p-1}{p}} \\
& \left(\int_{0}^{t} \theta^{p}(s) \Omega^{p}\left(\|x\|+\|x\|_{C} \|\right) \mathrm{d} s\right)^{\frac{1}{p}} \\
\leq & M\left(\|\phi(0)\|+e_{1}\right) \\
& +\frac{M T^{\alpha-\frac{1}{p}}\|\theta(s)\|_{p} \Omega\left(2 T^{\frac{1}{p}} k\right)}{\Gamma(\alpha)}\left(\frac{p-1}{p \alpha-1}\right)^{\frac{p-1}{p}} .
\end{aligned}
$$

for any $t \in[0, T]$. This means that $F W_{0} \subset W_{0}$.

Let

$W_{n+1}=F W_{n}$ for $n=0,1,2, \ldots$

Then

$F W_{n} \subset C([0, T], X)$,

is bounded and equicontinuous on [0, T]. Furthermore, $W_{n+1} \subset W_{n}$, because $W_{1} \subset W_{0}$. Hence,

$$
\begin{aligned}
\chi\left(W_{n+1}(t)\right) \leq & \chi\left(T(t)\left(x_{0}-g\left(W_{n}\right)\right)\right)+\chi\left(\frac{1}{\Gamma(\alpha)} \int_{0}^{t}(t-\eta)^{\alpha-1}\right. \\
& \left.T(t-\eta) f\left(\eta, W_{n}(\eta),\left(W_{n}\right)_{\eta}\right) \mathrm{d} \eta\right) \\
\leq & \chi\left(T(t)\left(x_{0}-g\left(W_{n}\right)\right)\right)+\frac{1}{\Gamma(\alpha)} \\
& \int_{0}^{t} \chi\left((t-\eta)^{\alpha-1} T(t-\eta) f\left(\eta, W_{n}(\eta),\left(W_{n}\right)_{\eta}\right)\right) \mathrm{d} \eta
\end{aligned}
$$




$$
\begin{aligned}
& \leq c_{1} \chi_{p}\left(W_{n}\right)+\frac{2}{\Gamma(\alpha)} \int_{0}^{t}(t-\eta)^{\alpha-1} c_{2}(\eta) \chi\left(W_{n}\right) \mathrm{d} \eta \\
& \leq\left(c_{1}+\frac{2\left\|c_{2}\right\|_{q} T^{\alpha-\frac{1}{p}}}{\Gamma(\alpha)}\left(\frac{p-1}{p \alpha-1}\right)^{\frac{p-1}{p}}\right) \chi_{p}\left(W_{n}\right),
\end{aligned}
$$

for $n=0,1,2, \ldots$ and a.e. $t \in[0, T]$. By Lemma 2.4, this implies that

$\chi_{p}\left(W_{n+1}\right) \leq T^{\frac{1}{p}}\left(c_{1}+\frac{2\left\|c_{2}\right\|_{q} T^{\alpha-\frac{1}{p}}}{\Gamma(\alpha)}\left(\frac{p-1}{p \alpha-1}\right)^{\frac{p-1}{p}}\right) \chi_{p}\left(W_{n}\right)$,

$$
\text { for } n=0,1,2, \ldots \text {. }
$$

Define

$$
\widehat{W}_{n}=L^{p}-\overline{\operatorname{conv}}\left(W_{n}\right), \text { for } n=0,1,2, \ldots
$$

Then $\widehat{W}_{n+1} \subset \widehat{W}_{n}$ because $W_{n+1} \subset W_{n}$, and furthermore one has

$$
\begin{aligned}
\chi_{p}\left(\widehat{W}_{n+1}\right) & =\chi_{p}\left(W_{n+1}\right) \\
& \leq T^{\frac{1}{p}}\left(c_{1}+\frac{2\left\|c_{2}\right\|_{q} T^{\alpha-\frac{1}{p}}}{\Gamma(\alpha)}\left(\frac{p-1}{p \alpha-1}\right)^{\frac{p-1}{p}}\right) \chi_{p}\left(W_{n}\right) \\
& =T^{\frac{1}{p}}\left(c_{1}+\frac{2\left\|c_{2}\right\|_{q} T^{\alpha-\frac{1}{p}}}{\Gamma(\alpha)}\left(\frac{p-1}{p \alpha-1}\right)^{\frac{p-1}{p}}\right) \chi_{p}\left(\widehat{W}_{n}\right),
\end{aligned}
$$

for $n=0,1,2, \ldots$ Lemma 2.1 shows that

$$
\widehat{W}=\cap_{n=1}^{\infty} \widehat{W}_{n},
$$

is nonempty, convex and compact in $L^{p}([0, T], X)$ and $F \widehat{W} \subset \widehat{W}$. Let

$$
U=\overline{\operatorname{conv}}(F \widehat{W}) \text {. }
$$

Then $U \subset C([0, T], X)$ and $F U \subset U$, since

$U=\overline{\operatorname{conv}}(F \widehat{W}) \subset \overline{\operatorname{conv}}(\widehat{W}) \subset L^{p}-\overline{\operatorname{conv}}(\widehat{W})=\widehat{W}$.

Now we prove that $U \subset C([0, T], X)$ is compact. First, by the hypothesis $\left(H_{1}\right)$ and Lemma $2.5, F \widehat{W}$ is equicontinuous on $[0, T]$, as

$$
g: L^{p}([0, T], X) \rightarrow X,
$$

is continuous and $\widehat{W} \subset L^{p}([0, T], X)$ is compact.

Furthermore,

$$
\begin{gathered}
\chi(\widehat{W}(t)) \leq \chi\left(T(t)\left(x_{0}-g(\widehat{W})\right)\right)+\chi\left(\frac{1}{\Gamma(\alpha)} \int_{0}^{t}(t-\eta)^{\alpha-1}\right. \\
\left.T(t-\eta) f\left(\eta, \widehat{W}(\eta), \widehat{W}_{\eta}\right) \mathrm{d} \eta\right)
\end{gathered}
$$

$$
\begin{aligned}
\leq & \chi\left(T(t)\left(x_{0}-g(\widehat{W})\right)\right)+\frac{1}{\Gamma(\alpha)} \\
& \int_{0}^{t} \chi\left((t-\eta)^{\alpha-1} T(t-\eta) f\left(\eta, \widehat{W}(\eta), \widehat{W}_{\eta}\right)\right) \mathrm{d} \eta \\
\leq & c_{1} \chi_{p}(\widehat{W})+\frac{2}{\Gamma(\alpha)} \int_{0}^{t}(t-\eta)^{\alpha-1} c_{2}(\eta) \chi(\widehat{W}) \mathrm{d} \eta \\
\leq & \left(c_{1}+\frac{2\left\|c_{2}\right\|_{q} T^{\alpha-\frac{1}{p}}}{\Gamma(\alpha)}\left(\frac{p-1}{p \alpha-1}\right)^{\frac{p-1}{p}}\right) \chi_{p}(\widehat{W})=0,
\end{aligned}
$$

for any $t \in[0, T]$. Hence

$$
F \widehat{W} \subset C([0, T], X),
$$

is precompact, and hence so is

$$
U \subset C([0, T], X) .
$$

The proof is complete by Schauder's fixed point theorem.

Remark 3.3 Without hypothesis $\left(H_{2}\right)$ the map $F$, defined above, may not be continuous from $L^{p}([0, T], X)$ to itself, since the operator $f$ may fail to be continuous under the growth condition (3.4) above. So we use the fixed point theorem on $C([0, T], X)$ rather than on $L^{p}([0, T], X)$, as $F$ is obviously continuous from $C([0, T], X)$ to itself.

\section{Applications}

In this section, we give an example to illustrate the above results.

Consider the following nonlinear fractional parabolic systems of the form

$$
\begin{gathered}
\frac{\partial^{\alpha}}{\partial t^{\alpha}} u(t, x)=\mu_{1} \Delta u(t, x)+F_{1}\left(t, u(t, x), u_{t}(x), v(t, x), v_{t}(x)\right), \\
t \in(0, T), x \in \Omega, \\
\frac{\partial^{\alpha}}{\partial t^{\alpha}} v(t, x)=\mu_{2} \Delta v(t, x)+F_{2}\left(t, u(t, x), u_{t}(x), v(t, x), v_{t}(x)\right), \\
t \in(0, T), x \in \Omega, \\
u(0, x)=\varphi_{1}+g_{1}(u(t, x), v(t, x)), \quad x \in \Omega, \\
v(0, x)=\varphi_{2}+g_{2}(u(t, x), v(t, x)), \quad x \in \Omega,
\end{gathered}
$$

where $\Omega$ is a bounded domain of $\mathbb{R}^{p}, p \geq 1$, with smooth boundary $\Gamma, \mu_{1}, \mu_{2}$ are positive constants,

$F_{1}, F_{2}: \mathbb{R} \times \mathbb{R} \times C([-q, 0], \mathbb{R}) \rightarrow \mathbb{R}$,

are given mappings. Here 
$F_{2}\left(t, u(t, x), u_{t}(x), v(t, x), v_{t}(x)\right)=\int_{\Omega} h_{1}\left(t, x, z, u(z), u_{t}(z)\right) \mathrm{d} z$,

$g_{2}(u(t, x), v(t, x))=\int_{\Omega} \int_{0}^{T} h_{2}(t, x, z, u(z)) \mathrm{d} t \mathrm{~d} z$.

Let

$X=L^{2}(\Omega) \times L^{2}(\Omega)$

be endowed with the inner product $\langle\cdot, \cdot\rangle$ defined by

$<(u, v),(\bar{u}, \bar{v})>=\left\langle u, \bar{u}>_{L^{2}(\Omega)}+<v, \bar{v}>_{L^{2}(\Omega)}\right.$,

for each $(u, v),(\bar{u}, \bar{v}) \in X$. Obviously $X$ is a separable real Hilbert space. Define $A: D(A) \subset X \rightarrow X$ given by

$A(u, v)=\left(\mu_{1} \Delta u, \mu_{2} \Delta v\right)$, for each $(u, v) \in D(A)$

with the domain

$D(A)=\left\{(u, v) \in X: \frac{\partial^{\alpha}}{\partial t^{\alpha}} u, \frac{\partial^{\alpha}}{\partial t^{\alpha}} v \in X, \Delta u, \Delta v \in X\right\}$.

Now define

$F:[0, T] \times X \rightarrow X$ and $g: C([0, T], X) \rightarrow X$

by

$$
\begin{aligned}
F\left(t,(u, v),\left(u_{t}, v_{t}\right)\right)= & \left(F_{1}\left(t, u(t, x), u_{t}(x), v(t, x), v_{t}(x)\right),\right. \\
& \left.F_{2}\left(t, u(t, x), u_{t}(x), v(t, x), v_{t}(x)\right)\right), \\
g(t,(u, v))= & \left(g_{1}\left(t,(u, v),\left(u_{t}, v_{t}\right)\right), g_{2}\left(t,(u, v),\left(u_{t}, v_{t}\right)\right)\right),
\end{aligned}
$$

for

$(u, v) \in X,\left(u_{t}, v_{t}\right) \in C([-q, 0], X) \times C([-q, 0], X)$,

where $F_{i}$ and $g_{i}$ are superposition mappings associated with $F_{i}$ and $g_{i}$ defined by

$$
\begin{aligned}
& F_{i}(t,(u, v))=\left\{h \in L^{2}(\Omega), h(x)=F_{i}(t,(u(x), v(x)),\right. \\
& \left.\left.\quad\left(u_{t}(x), v_{t}(x)\right)\right), \quad \text { a.e. for } x \in \Omega\right\}, \\
& g_{i}(t,(u, v))=\left\{h \in L^{2}(\Omega), h(x)=g_{i}((u(x), v(x)),\right. \\
& \left.\left.\quad\left(u_{t}(x), v_{t}(x)\right)\right), \quad \text { a.e. for } x \in \Omega\right\} .
\end{aligned}
$$

Observe that Eq.(4.1) may be rewritten as

$$
\begin{aligned}
\frac{\mathrm{d}^{\alpha} \mathrm{U}}{\mathrm{d} t^{\alpha}} & =A u+F\left(t, U(t), U_{t}\right), \quad t \in(0, T), \quad \text { a. e. } \\
U(0) & =\varphi+g(U)
\end{aligned}
$$

where

$$
U(t)=(u(t), v(t)), \quad \varphi=\left(\varphi_{1}, \varphi_{2}\right)
$$

while $A, F$ and $g$ are as above.

Suppose that:
(1) There exists $k_{2}(t) \in L(0, T)$ such that $F_{1}:[0, T] \times$ $\mathbb{R} \rightarrow \mathbb{R}$ is a Carathédory-type function and, for $u, u^{\prime}, v, v^{\prime} \in \mathbb{R}$,

$$
\left|F_{1}(t, u, v)-F_{1}\left(t, u^{\prime}, v^{\prime}\right)\right| \leq k_{2}(t)\left(\left|u-u^{\prime}\right|_{2}+\left|v-v^{\prime}\right|^{2}\right) .
$$

(2) There exists a constant $k_{1}$ such that $g_{1}: \mathbb{R} \rightarrow \mathbb{R}$ is a Carathédory-type function and, for $u, u^{\prime}, v, v^{\prime} \in \mathbb{R}$,

$$
\left|g_{1}(u, v)-g_{1}\left(u^{\prime}, v^{\prime}\right)\right| \leq k_{1}\left(\left|u-u^{\prime}\right|_{2}+\left|v-v^{\prime}\right|^{2}\right) \text {. }
$$

(3) For $i=1,2, h_{i}:[0, T] \times \Omega \times \Omega \times \mathbb{R} \times \mathbb{R} \rightarrow \mathbb{R}$ satisfies Carathédory conditions. In addition:

(i)

$\left|h_{i}(t, x, z, r, s)-h_{i}\left(t, x^{\prime}, z, r, s\right)\right| \leq \omega_{i}^{k}\left(t, x, x^{\prime}, z\right)$,

for $(t, x, z),\left(t, x^{\prime}, z\right) \in[0, T] \times \Omega \times \Omega$ and $|r|,|s| \leq k$, where $\omega_{i}^{k} \in L\left([0, T] \times \Omega^{3}\right)$ are such that

$\lim _{x^{\prime} \rightarrow x} \int_{\Omega} \int_{0}^{T} \omega_{i}^{k}\left(t, x, x^{\prime}, z\right) \mathrm{d} t \mathrm{~d} z=0$,

uniformly for $x \in \Omega, i=1,2$, and for $t \in[0, T]$

$\lim _{x^{\prime} \rightarrow x} \int_{\Omega} \omega_{i}^{k}\left(t, x, x^{\prime}, z\right) \mathrm{d} z=0$,

uniformly for $x \in \Omega$;

$\mid h_{i}\left(t, x, z, r, s \mid \leq \rho_{i}(t)\left(|r|^{2}+|s|^{2}\right)^{\frac{1}{2}}+\omega_{i}(t, x, z)\right.$,

where $\rho_{i} \in L(0, T)$ and

$$
\begin{aligned}
\delta_{i} & =\int_{0}^{T} \int_{\Omega \times \Omega}\left(\omega_{i}(t, x, z)\right)^{2} \mathrm{~d} x \mathrm{~d} z \mathrm{~d} t \\
& <+\infty, \quad i=1,2 .
\end{aligned}
$$

Adapting the arguments given in [47] it is not difficult to show that $g$ satisfies the hypothesis $\left(\mathrm{H}_{3}\right)$ with

$$
\begin{gathered}
c_{1}=k_{1}, \quad d_{1}^{2}=k_{1}^{2}+2 m(\Omega)\left\|\rho_{1}\right\|_{1}^{2}, \\
e_{1}^{2}=2 m(\Omega) \delta_{1},
\end{gathered}
$$

and $f$ satisfies the hypothesis $\left(\mathrm{H}_{2}\right)$ with

$$
\begin{gathered}
c_{2}(t)=k_{2}(t), \quad d^{2}(t)=k_{2}^{2}(t)+2 m(\Omega) \rho_{2}^{2}(t), \\
\left\|e_{2}\right\|_{1}^{2}=2 m(\Omega) \delta_{2},
\end{gathered}
$$

where $m(\Omega)$ means the Lebesgue measure of $\Omega$ in $\mathbb{R}^{p}$. Using Corollary 3.1 , we conclude that Eq.(4.1) has at least one generalized solution 


$$
\left(u_{1}, u_{2}\right) \in C\left([0, T], L^{2}(\Omega) \times L^{2}(\Omega)\right),
$$

provided that

$$
\begin{aligned}
& M\left(k_{1}^{2}+2 m(\Omega)\left\|\rho_{1}\right\|_{1}^{2}\right)^{\frac{1}{2}} T^{\frac{1}{p}}+\frac{4 M T^{\alpha}}{\Gamma(\alpha)}\left(\frac{p-1}{p \alpha-1}\right)^{\frac{p-1}{p}} \\
& \left(\int_{0}^{T}\left(k_{2}^{2}(t)+2 m(\Omega) \rho_{2}^{2}(t)\right)^{\frac{p}{2}} \mathrm{~d} t\right)^{\frac{1}{p}}<1,
\end{aligned}
$$

and

$$
T^{\frac{1}{p}}\left(k_{1}+\frac{2\left\|k_{2}\right\|_{q} T^{\alpha-\frac{1}{p}}}{\Gamma(\alpha)}\left(\frac{p-1}{p \alpha-1}\right)^{\frac{p-1}{p}}\right)<1 .
$$

Acknowledgments This work is supported by the National Natural Science Foundation of China (No.11301090), Appropriative Researching Fund for Professors and Doctors, Guangdong University of Education (No. 2013ARF02).

Open Access This article is distributed under the terms of the Creative Commons Attribution 4.0 International License (http:// creativecommons.org/licenses/by/4.0/), which permits unrestricted use, distribution, and reproduction in any medium, provided you give appropriate credit to the original author(s) and the source, provide a link to the Creative Commons license, and indicate if changes were made.

\section{References}

1. Ahn, V., McVinisch, R.: Fractional differential equations driven by Lévy noise. J. Appl. Math. Stoch. Anal. 16, 97-119 (2003)

2. D. Benson, The Fractional Advection-Dispersion Equation, Ph.D. Thesis, University of Nevada, Reno, NV, 1998

3. Schumer, R., Benson, D.: Eulerian derivative of the fractional advection-dispersion equation. J. Contam. Hydrol. 48, 69-88 (2001)

4. Sayed, A.: Nonlinear functional-differential equations of arbitrary orders. Nonlinear Anal. Theory Methods Appl. 33, 181-186 (1998)

5. Ling, Y., Ding, S.: A class of analytic functions defined by fractional derivation. J. Math. Anal. Appl. 186, 504-513 (1994)

6. N'Guérékata, G.: Cauchy problem for some fractional abstract differential equation with nonlocal conditions. Nonlinear Anal. Theory Methods Appl. 70, 1873-1876 (2009)

7. Lahshmikantham, V., Devi, J.: Theory of fractional differential equations in Banach spaces. Eur. J. Pure Appl. Math. 1, 38-45 (2008)

8. Glockle, W., Nonnemacher, T.: A fractional calculus approach of self-similar protein dynamics. Biophys. J. 68, 46-53 (1995)

9. Metzler, F., Schick, W., Kilian, H., Nonnemacher, T.: Relaxation in filled polymers: A fractional calculus approach. J. Chem. Phys. 103, 7180-7186 (1995)

10. Ahmad, B., Sivasundaram, S.: Some existence results for fractional integrodifferential equations with nonlinear conditions. Commmun. Math. Anal. 12, 107-112 (2008)

11. Bhaskar, T., Lakshmikantham, V., Leela, S.: Fractional differential equations with Krasnoselskii-Krein-type condition. Nonlinear Anal. Hybrid Syst. 3, 734-737 (2009)

12. Lakshmikantham, V., Leela, S.: Nagumo-type uniqueness result for fractional differential equations. Nonlinear Anal. Theory Methods Appl. 71, 2886-2889 (2009)
13. Anguraj, A., Karthikeyan, P., N'Guérékata, G.: Nonlocal Cauchy problem for some fractional abstract integro-differential equations in Banach spaces. Commmun. Math. Anal. 6, 31-35 (2009)

14. Cao, J., Yang, Q., Huang, Z.: Existence of anti-periodic mild solutions for a class of semilinear fractional differential equations. Commun. Nonlinear Sci. Numer. Simulat. 17, 277-283 (2012)

15. Cao, J., Yang, Q., Huang, Z.: Optimal mild solutions and weighted pseudo-almost periodic classical solutions of fractional integro-differential equations. Nonlinear Anal. Theory Methods Appl. 74, 224-234 (2011)

16. Rida, S., Sayed, A., Arafa, A.: On the solutions of time-fractional reaction-diffusion equations. Commun. Nonlinear Sci. Numer. Simulat. 15, 3847-3854 (2010)

17. Wang, R., Chen, D., Xiao, T.: Abstract fractional Cauchy problems with almost sectorial operators. J. Differ. Equations 252, 202-235 (2012)

18. Obukhovskii, V., Yao, J.: Some existence results for fractional functional differential equations. Fixed Point Theory 11, 85-96 (2010)

19. Eidelman, S., Kochubei, A.: Cauchy problem for fractional diffusion equations. J. Differ. Equations 199, 211-255 (2004)

20. Balachandran, K., Kiruthika, S., Trujillo, J.: Existence results for fractional impulsive integrodifferential equations in Banach spaces. Commun. Nonlinear Sci. Numer. Simulat. 16, 1970-1977 (2011)

21. Wang, J., Zhou, Y., Wei, W.: A class of fractional delay nonlinear integrodifferential controlled systems in Banach spaces. Commun. Nonlinear Sci. Numer. Simulat. 16, 4049-4059 (2011)

22. Agrawal, O.: Solution for a fractional diffusion-wave equation defined in a bounded domain. J. Nonlinear Dynam. 29, 145-155 (2002)

23. Liang, J., Yang, H.: Controllability of fractional integro-differential evolution equations with nonlocal conditions. Appl. Math. Comput. 254, 20-29 (2015)

24. Zhang, X.: Positive solutions for a class of singular fractional differential equation with infinite-point boundary value conditions. Appl. Math. lett. 39, 22-27 (2015)

25. Agarwal, R., Lupulescu, V., O'Regan, D., Rahman, G.: Fractional calculus and fractional differential equations in nonreflexive Banach spaces. Commun. Nonlinear Sci. Numer. Simulat. 20, 59-73 (2015)

26. Henderson, J., Luca, R.: Positive solutions for a system of fractional differential equations with coupled integral boundary conditions. Appl. Math. Comput. 249, 182-197 (2014)

27. Wang, R., Xiang, Q., Zhu, P.: Existence and approximate controllability for systems governed by fractional delay evolution inclusions. Optimization 63, 1191-1204 (2014)

28. Wang, R., Yang, Y.: On the Cauchy problems of fractional evolution equations with nonlocal initial conditions. Results. Math. 63, 15-30 (2013)

29. Wang, R., Xiang, Q., Zhou, Y.: Fractional delay control problems: Topological structure of solution sets and its applications. Optimization 63, 1249-1266 (2014)

30. Zhou, Y., Jiao, F.: Existence of mild solutions for fractional neutral evolution equations. Comput. Math. Appl. 59, 1063-1077 (2010)

31. Bëleanu, D., Mustafa, O.: On the global existence of solutions to a class of fractional differential equations. Comput. Math. Appl. 59, 1835-1841 (2010)

32. Mophou, G., N'Guérékata, G.: Existence of the mild solution for some fractional differential equations with nonlocal conditions. Semigroup Forum 79, 315-322 (2009)

33. Li, C., Luo, X., Zhou, Y.: Existence of positive solutions of the boundary value problem for nonlinear fractional differential equations. Comput. Math. Appl. 59, 1363-1375 (2010) 
34. Zhang, S.: Positive solutions to singular boundary value problem for nonlinear fractional differential equation. Comput. Math. Appl. 59, 1300-1309 (2010)

35. Agarwal, R., Zhou, Y., He, Y.: Existence of fractional neutral functional differential equations. Comput. Math. Appl. 59, 1095-1100 (2010)

36. Podlubny, I.: Fractional Differential Equations. Academic Press, San Diego (1999)

37. J. Banaś, K. Goebel, Measures of Noncompactness in Banach Spaces, Lect. Notes Pure Appl. Math., vol. 60, Dekker, New York, 1980

38. Darbo, G.: Punti unitti in transformazioni a condominio non compatto. Rend. Semin. Mat. Univ. Padova 24, 84-92 (1955)

39. Sadovski, B.: On a fixed point principle. Funct. Anal. Appl. 1, 74-76 (1967)

40. Istratescu, V.: Fixed Point Theory. Reidel, Boston (1981)

41. Hajji, A., Hanebaly, E.: Commuting mappings and -compact type fixed point theorems in locally convex spaces. Int. J. Math. Anal. 1, 661-680 (2007)
42. Hajji, A.: A generalization of Darbos fixed point and common solutions of equations in Banach spaces. Fixed Point Theory and Applications 2013, 62 (2013)

43. Kisielewicz, M.: Multivalued differential equations in separable Banach spaces. J. Optim. Theory Appl. 37, 231-249 (1982)

44. Xue, X.: $L^{p}$ theory for semilinear nonlocal problems with measure of noncompactness in separable Banach spaces. J. Fixed Point Theory Appl. 5, 129-144 (2009)

45. Barbu, V.: Nonlinear Semigroups and Differential Equations in Banach Spaces. Noordhoff, Leyden (1976)

46. Pavel, N.: Nonlinear evolution operators and semigroup. Lecture Notes in Math, vol. 1260. Springer-Verlag, New York (1987)

47. Martin, R.: Nonlinear Operators and Differential Equations in Banach Spaces. Wiley, New York (1976) 